\title{
Burden of symptoms associated with development of metastatic bone disease in patients with breast cancer
}

\author{
Charles Cleeland $^{1} \cdot$ Roger von Moos $^{2} \cdot$ Mark S. Walker $^{3} \cdot$ Yuanyuan Wang ${ }^{4}$. \\ Jianqing $\mathrm{GaO}^{4}$ • Mariana Chavez-MacGregor ${ }^{1}$ • Alexander Liede ${ }^{5}$ • Jorge Arellano ${ }^{6}$. \\ Arun Balakumaran ${ }^{6}$ Yi Qian ${ }^{6}$
}

Received: 9 September 2015 / Accepted: 7 March 2016/Published online: 29 March 2016

(C) The Author(s) 2016. This article is published with open access at Springerlink.com

\begin{abstract}
Purpose Women with breast cancer frequently develop painful bone metastases. This retrospective study was designed to longitudinally characterize patterns of patient-reported symptoms among patients with breast cancer relative to the diagnosis of bone metastases.

Methods Patient records were identified from the Oncology Services Comprehensive Electronic Records (OSCER) database which includes outpatient oncology practices across the USA. Symptom burden was assessed by Patient Care Monitor (PCM) assessments, which are administered as part of routine care in a subset of these practices. Eligible patients were women diagnosed with breast cancer (ICD-9-CM 174.xx) who developed bone metastases (ICD-9-CM 198.5) and had $\geq 1$
\end{abstract}

This study was presented at the 36th Annual San Antonio Breast Cancer Symposium, December 10-14, 2013

Electronic supplementary material The online version of this article (doi:10.1007/s00520-016-3154-x) contains supplementary material, which is available to authorized users.

Charles Cleeland

ccleeland@mdanderson.org

1 Department of Symptom Research, MD Anderson Cancer Center, University of Texas, 1515 Holcombe Boulevard, Unit Number: 1450, Room Number: FCT11.5064, Houston, TX 77030, USA

2 Kantonsspital Graubünden, Chur, Switzerland

3 Vector Oncology, Memphis, TN, USA

4 IMS Health, Plymouth Meeting, PA, USA

5 Amgen Inc., South San Francisco, CA, USA

6 Amgen Inc., Thousand Oaks, CA, USA
PCM assessment between January 2007 and December 2012. The pre-specified endpoint was the occurrence of moderate to severe symptom burden, defined as PCM score $\geq 4$ (010 scale).

Results One thousand one hundred five women (median age, 61) met the eligibility criteria. Worsening of symptoms, particularly fatigue and pain, occurred in the months leading up to the diagnosis of bone metastases. After bone metastases diagnosis, the rate of increase in the proportion of patients experiencing moderate/severe symptoms slowed, but continued to climb during followup. Median time to moderate/severe symptoms was 0.9 month for fatigue, 1 month for pain, 2.9 months for trouble sleeping, and 7.7 months for numbness/tingling. Half of the patients received bone-targeted agents after diagnosis of bone metastases.

Conclusions Symptom burden, especially pain and fatigue, increased both before and after the diagnosis of bone metastases, highlighting the need for proactive monitoring and management of symptoms in breast cancer patients.

Keywords Metastatic breast cancer - Bone metastases · Symptom burden $\cdot$ Pain $\cdot$ Bone-targeting agents

\section{Introduction}

Over $70 \%$ of women with advanced breast cancer will eventually develop metastases to the bone, the most common site of distant metastasis in this population [1]. The consequences of bone metastases are severe and include pain, skeletal-related events (pathological fracture, spinal cord compression, and the need for surgery or radiotherapy to bone), and decreased health-related quality of life 
(HRQoL). Bone pain is usually the first symptom of bone metastases, leading to their diagnosis and subsequent management. Treatments such as bone-targeted agents (intravenous bisphosphonates [zoledronic acid and pamidronic acid] or denosumab) can reduce pain and skeletal-related events in patients with bone metastases [2-4]. Analgesic medication and external beam radiation therapy are also frequently used in the management of pain in these patients $[5,6]$.

Understanding the symptoms associated with the development of bone metastases might provide information leading to better management of patients with advanced breast cancer. Pain, fatigue, depression, distress, anxiety, disturbed sleep, dry mouth, numbness/tingling, and gastrointestinal symptoms have been identified as key problems in this population [7-10]. In the Eastern Cooperative Oncology Group (ECOG) Symptom Patterns and Practice (SOAPP) study [7], which used the M.D. Anderson Symptom Inventory (MDASI) [11] to evaluate symptoms, the seven most prevalent moderate to severe symptoms in 1544 patients with breast cancer were identified as (in order from highest to lowest prevalence): fatigue/tiredness, disturbed sleep, pain, dry mouth, distress, numbness tingling, and sadness. Studies examining symptom-related outcomes in women diagnosed with metastatic breast cancer have shown that cancer-related symptoms increased over time along with disease progression [12] and that increases in depression and pain predicted increases in trouble sleeping [13].

As bone metastases are often initially asymptomatic, they may not be diagnosed and treated until bone pain or skeletal complications have occurred; therefore, damage to the bone structure may already be substantial, although this has yet to be proven. Prospective data on the changes in symptom burden in relation to the diagnosis of bone metastases may reveal clues to earlier diagnosis and management. There are few longitudinal data on symptom burden in women with metastatic breast cancer [12-14], and none of these studies evaluated symptom trajectory for individual symptoms with reference to the diagnosis or onset of bone metastases. We hypothesized that pain and other symptoms may increase before a diagnosis of bone metastases is made.

Using real-world data from electronic health records of patients from oncology practices in the USA, this retrospective study was designed to longitudinally characterize patterns of patient-reported symptoms among patients with breast cancer relative to the diagnosis of bone metastases. The primary objective was to characterize patient-reported symptoms over time using data collected from the Patient Care Monitor (PCM) instrument, while pain management trends and use of bone-modifying agents were exploratory objectives.

\section{Methods}

\section{Eligible patients}

Patient records were identified from the Oncology Services Comprehensive Electronic Records (OSCER) database, which includes data from outpatient oncology/hematology practices across the USA [15]. Over a 7-year data span (2005-2012), these databases contained information on approximately 560,000 patients from over 65 communitybased or hospital-affiliated oncology/hematology practices in over 35 states. The Vector Oncology data is a subset of OSCER including ten oncology practices. Participating practices are located in the Northwest, Midwest, and Northeast USA, but the majority of patients are from practices in the South USA. Practices range in size from 2 to more than 20 physicians, are rural and urban, and are not all members of any one group purchasing organization. Eligible patients were women diagnosed with invasive breast cancer (ICD-9-CM 174.xx) who developed bone metastases (ICD-9-CM 198.5) during their care and had $\geq 1$ Vector Oncology PCM assessment between January 2007 and October 2012. During the study period, three bone-targeted therapies were available for the prevention of skeletal-related events for breast cancer patients with bone metastases: two intravenous bisphosphonates (zoledronic acid [Zometa $\left.{ }^{\circledR}\right]$ and pamidronic acid $\left[\right.$ Aredia $\left.^{\circledR}\right]$ ) and denosumab (XGEVA ${ }^{\circledR}$ ), a fully human monoclonal antibody against RANKL, approved in the USA since November 2010.

\section{Patient-reported outcome measure}

The PCM version 2.0 is a validated instrument for summarizing oncology-related patient symptoms [16-18]. Patients complete the PCM surveys typically prior to their office visit or before a chemotherapy infusion using a touch screen tablet personal computer (requires 10-12 min to complete). The PCM assesses 86 individual symptoms and generates six indices that describe global function, including general physical symptoms, treatment side effects, despair, acute distress, impaired ambulation, and impaired performance. Patients were asked to rate symptoms on an 11-point scale: $0=$ not a problem to $10=$ as bad as possible. Moderate or severe symptoms were defined by a PCM score $\geq 4$ based on an analysis of Brief Pain Inventory-Short Form (also an 11-point Likert scale) results [19]. The six PCM items selected for this study (fatigue, trouble sleeping, physical pain, anxious, numbness/tingling, and loss of interest in others) were based on the subset of symptoms previously identified as the seven most prevalent moderate to severe symptoms in the ECOG SOAPP study (in order from highest to lowest prevalence: fatigue/tiredness, disturbed sleep, pain, dry mouth, distress, numbness/tingling, and sadness) [7]. As the PCM data available to us did not 
contain the item dry mouth, we could not evaluate this symptom. To evaluate distress, we selected the PCM item "anxious, " and to evaluate sadness, we selected the PCM item "loss of interest in others," which is a measure of depression [18].

\section{Endpoints}

The pre-specified endpoint in this study was a PCM score representing moderate to severe symptom burden (PCM score of $\geq 4$ ).

\section{Statistical analyses}

Analyses were conducted relative to two different definitions: (1) the recorded diagnosis of bone metastases (Fig. 1) and (2) the onset of bone metastases (Fig. 2), which was established as 1 month prior to the recorded diagnosis date, because we noted that the investigation and subsequent diagnosis of bone metastasis is typically triggered by an increase in symptoms, indicating that the actual onset occurred earlier.

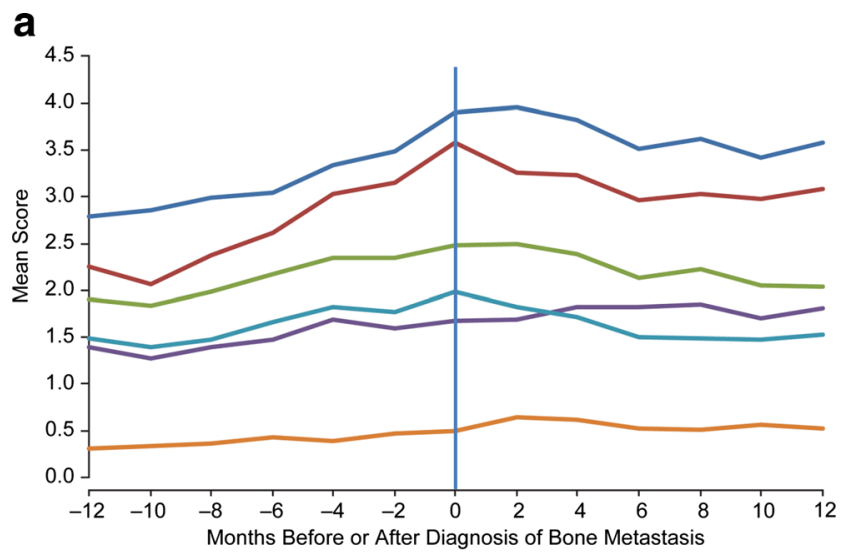

b

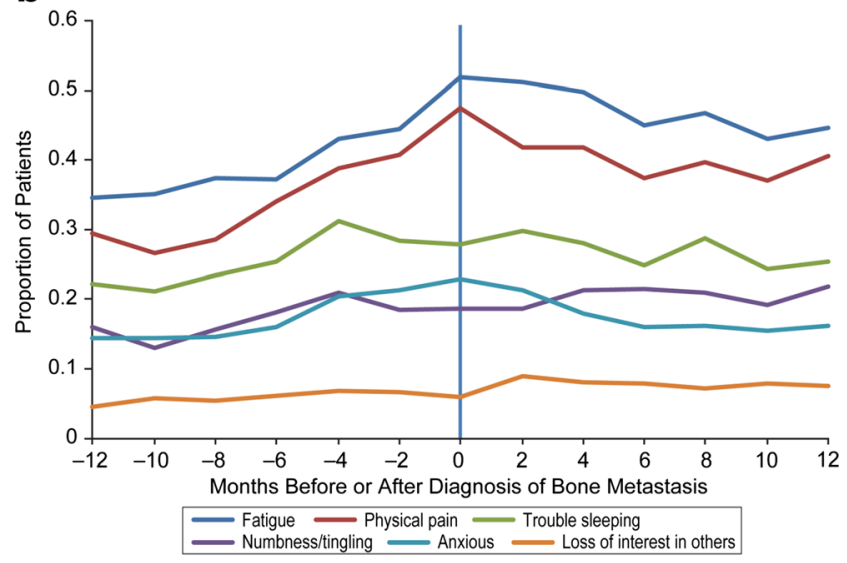

Fig. 1 Symptom burden over time relative to the diagnosis of bone metastases. a Mean PCM score over time. b Proportion of patients with moderate or severe symptoms (PCM score $\geq 4$ ). The number of patients assessed at each time point and for each PCM item is shown in Supplemental Table S1
Mean PCM score and proportion of patients with moderate to severe symptom burden were reported by 2 -month intervals during the year before and after the diagnosis of bone metastases; missing PCM values were not imputed. A generalized linear mixed model was used to evaluate symptom progression before and after diagnosis of bone metastasis.

Kaplan-Meier methods were used to estimate the time to development of moderate to severe symptoms after the onset of bone metastases. Patients had to have at least 1 PCM record from the onset of diagnosis of bone metastases to 7 months after $(n=822)$, thereby excluding patients whose first PCM record was obtained more than 6 months after the onset of bone metastases. Patients with PCM score $\geq 4$ at the onset of bone metastases or in the prior 3 months were represented as having the symptom at time 0 .

Cox proportional hazards models, with factors defined a priori based on clinical judgement and expert opinion, were used to model effects on specific symptoms in multivariate analysis. Baseline factors in the model included race, age, visceral metastases, bone-targeted agent treatment, prior taxane therapy (before diagnosis of bone metastases), chemotherapy (after diagnosis of bone metastases), brain metastases, liver metastases, and lung metastases. Symptoms were modeled individually.

\section{Results \\ Patients}

A total of 1105 women met the eligibility criteria. Patients had a median age of 61 (range, 52-70); $57 \%$ were Caucasian and $23 \%$ were African-American (Table 1). Slightly over half ( $55 \%$ ) had metastases to the bone only, while $45 \%$ also had metastases to another site (25\% liver, $18 \%$ lung, and $14 \%$ brain). Data were not collected on which site of metastases was the first to be diagnosed. For those who had a stage recorded in the electronic medical record $(n=749), 307(49 \%)$ had stage IV disease as the earliest stage recorded. We note that the stage distribution does not reflect a typical distribution at diagnosis because patients had to have bone metastases to be included in the study. The median time from cancer diagnosis to diagnosis of bone metastases was 20.5 months. Fifteen percent had breast cancer and bone metastases diagnoses on the same day, and another $10 \%$ had the bone metastases diagnosis within 30 days of the original cancer diagnosis.

\section{Analgesic medication and bone-targeted agents}

No substantial increase in analgesic medication use was observed before vs after diagnosis of bone metastases, with $30 \%$ receiving prescriptions for pain medications in the 
a Fatigue

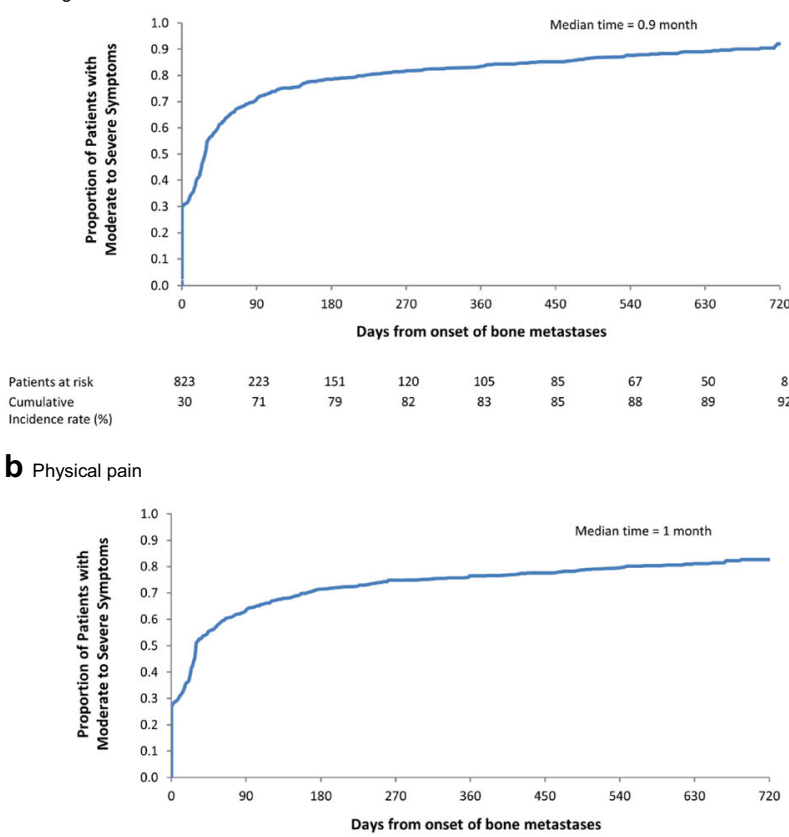

\section{Patients at risk} Cumulative

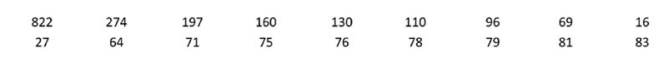

C Trouble sleeping

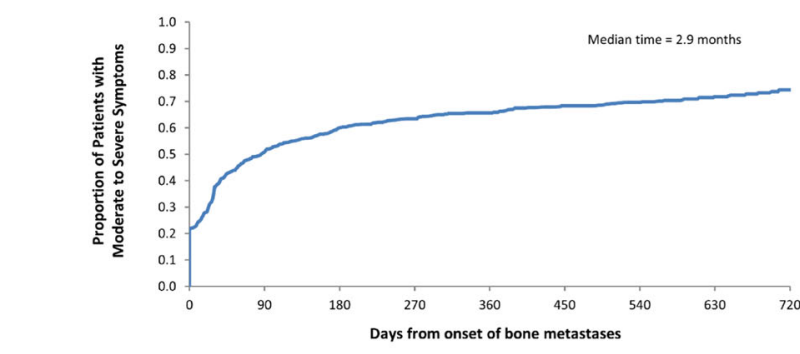

Patients at risk Cumulative d Numbness/tingling

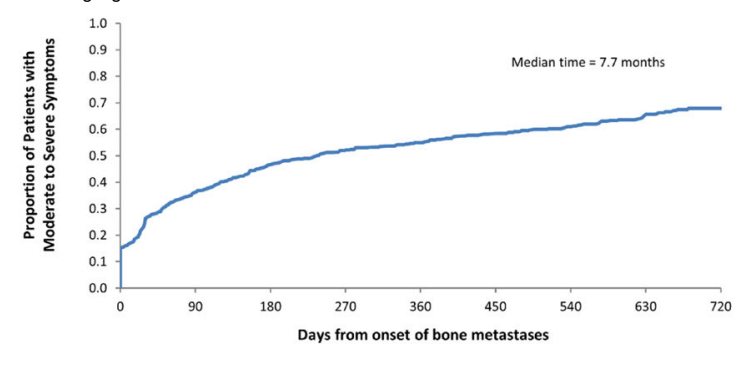

$\begin{array}{lccccccccc}\text { Patients at risk } & 823 & 458 & 346 & 277 & 238 & 194 & 157 & 117 & 24 \\ \text { Cumulative } & 15 & 36 & 47 & 52 & 55 & 58 & 61 & 66 & 68\end{array}$

e Anxiety

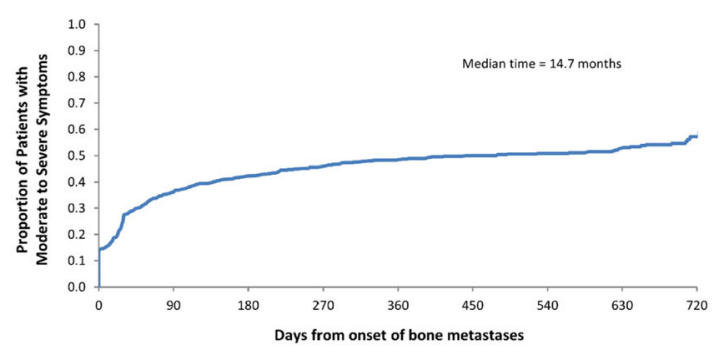

$\begin{array}{lccccccccc}\text { Patients at risk } & 819 & 449 & 362 & 305 & 255 & 216 & 185 & 140 & 26 \\ \text { Cumulative } & 14 & 36 & 42 & 46 & 49 & 50 & 51 & 53 & 57\end{array}$

f Loss of interest in others

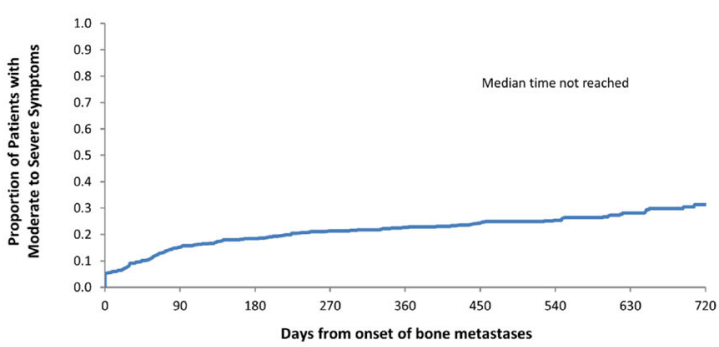

$\begin{array}{lccccccccc}\text { Patients at risk } & 819 & 596 & 498 & 425 & 361 & 301 & 256 & 190 & 34 \\ \text { Cumulative } & 5 & 15 & 19 & 21 & 23 & 24 & 25 & 28 & 31\end{array}$

Fig. 2 Kaplan-Meier estimated proportion of patients with moderate to severe symptoms over time. a Fatigue. b Physical pain. $\mathbf{c}$ Trouble sleeping. d Numbness/tingling. e Anxious. f Loss of interest in others

6 months before diagnosis of bone metastases compared with $34 \%$ after the diagnosis (Table 1). Similarly, $10 \%$ of patients were receiving strong opioid medications before the diagnosis compared with $7 \%$ after. A total of 182 patients ( $16 \%)$ were either new to pain medication or increased the strength of their prior pain medication after diagnosis of bone metastases. We note that an underestimation of analgesic use was anticipated in this study because the oncology patient record, despite capturing details of medications administered or prescribed in the oncology clinic, may not reliably capture those medications obtained over the counter or prescribed by another physician or at a different center, such as in the hospital.

Half of the patients $(n=527 ; 48 \%)$ were receiving bone-targeted therapy after the diagnosis of bone metastases. Most patients received bisphosphonates $(n=469)$ and some received the RANK ligand inhibitor, denosumab $(n=70)$; two patients received both. The median (Q1, Q3) time to use of bone-targeted therapy after diagnosis of bone metastases was $43(25,152)$ days. In the first year after the diagnosis of bone metastases, 772 patients $(70 \%)$ were on either bone-targeted agents and/ or chemotherapy (endocrine therapy is not included): $43 \%$ of these patients were on both, $39 \%$ were on bone-targeted agents only, and $18 \%$ were on chemotherapy only. Of the 611 patients on chemotherapy after diagnosis of bone metastases in the first year, $21 \%$ had chemotherapy both 6 months before and 6 months after the diagnosis. Of these, $49 \%$ remained on the same chemotherapy in both the preand post-period. 
Table 1 Patient characteristics

\begin{tabular}{|c|c|}
\hline Characteristic & $n=1105$ \\
\hline Median (range) age, years & $61(52,70)$ \\
\hline \multicolumn{2}{|l|}{ Race, $n(\%)$} \\
\hline Caucasian & $624(57 \%)$ \\
\hline African-American & $256(23 \%)$ \\
\hline Hispanic & $4(<1 \%)$ \\
\hline Asian & $3(<1 \%)$ \\
\hline Other & $218(20 \%)$ \\
\hline Months from breast cancer diagnosis to bone metastases, median (Q1, Q3) & $20.5(1.0,57.8$ \\
\hline \multicolumn{2}{|l|}{ Cancer stage (earliest recorded in CRF), $n(\%)$} \\
\hline Number of patients with available stage & 749 \\
\hline Stage 1 & $115(15 \%)$ \\
\hline Stage 2 & $190(25 \%)$ \\
\hline Stage 3 & $137(18 \%)$ \\
\hline Stage 4 & $307(41 \%)$ \\
\hline \multicolumn{2}{|l|}{ Location of metastases, $n(\%)$} \\
\hline Bone only & $605(55 \%)$ \\
\hline Bone and other site(s) & $500(45 \%)$ \\
\hline Liver & $272(25 \%)$ \\
\hline Lung & $195(18 \%)$ \\
\hline Brain & $152(14 \%)$ \\
\hline \multirow[t]{2}{*}{ Other } & $189(17 \%)$ \\
\hline & $n=1105$ \\
\hline \multicolumn{2}{|l|}{ Treatments received before diagnosis of bone metastases, $n(\%)$} \\
\hline Chemotherapy or biologic agents & $616(56 \%)$ \\
\hline Taxanes & $429(39 \%)$ \\
\hline Biologic agents & $129(12 \%)$ \\
\hline Hormonal therapies & $457(41 \%)$ \\
\hline \multicolumn{2}{|l|}{ Pain medication use } \\
\hline Before diagnosis of bone metastases $^{\mathrm{a}}$ & $336(30 \%)$ \\
\hline Non-opioid ${ }^{\mathrm{b}}$ & $57(5 \%)$ \\
\hline Opioid medication for moderate pain ${ }^{\mathrm{c}}$ & $31(3 \%)$ \\
\hline Strong opioid medications ${ }^{\mathrm{d}}$ & $109(10 \%)$ \\
\hline After diagnosis of bone metastases & $379(34 \%)$ \\
\hline Non-opioid ${ }^{\mathrm{b}}$ & $73(7 \%)$ \\
\hline Opioid medication for moderate pain ${ }^{\mathrm{c}}$ & $50(5 \%)$ \\
\hline Strong opioid medications ${ }^{\mathrm{d}}$ & $74(7 \%)$ \\
\hline $\begin{array}{l}\text { Increase in medication strength or new medication use from before to after } \\
\text { diagnosis of bone metastases }\end{array}$ & $182(16 \%)$ \\
\hline Non-opioid ${ }^{\mathrm{b}}$ & $30(3 \%)$ \\
\hline Opioid medication for moderate pain ${ }^{\mathrm{c}}$ & $22(2 \%)$ \\
\hline Strong opioid medications ${ }^{\mathrm{d}}$ & $54(5 \%)$ \\
\hline Bone-targeted agents received after diagnosis of bone metastases, $n(\%)$ & $527(48 \%)$ \\
\hline
\end{tabular}

${ }^{\text {a }}$ Medication use was collected for the 6 months before the diagnosis of bone metastases

${ }^{\mathrm{b}}$ Non-opioid medications included acetaminophen (ASA), aspirin, ibuprofen, and naproxen

${ }^{\mathrm{c}}$ Opioid medications for moderate pain included ASA-codeine, ASA/oxycodone, aspirin/oxycodone, and ASA/ hydrocodone

${ }^{\mathrm{d}}$ Strong opioid medications included morphine sulfate, hydromorphone, and fentanyl 


\section{Symptom burden}

Worsening of symptoms, as measured by PCM scores, occurred in the 2 to 4 months leading up to the diagnosis of bone metastases (Fig. 1a). Fatigue and pain, in particular, rose sharply prior to the diagnosis. The proportion of patients with moderate to severe symptoms also rose prior to diagnosis (Fig. 1b). The estimated increase in risk per month that patients would develop moderate to severe symptoms leading up to diagnosis was $9 \%(P<0.001)$ for both fatigue and pain and $19 \%(P<0.001)$ for numbness/tingling. For the other symptoms, change in risk over time of developing moderate to severe symptoms prior to bone metastases was not statistically significant (trouble sleeping, $3 \%, P=0.1$; anxiety, $0 \%$, $P=0.8$; loss of interest in others, $17 \%, P=0.2$ ). After the diagnosis of bone metastases, symptom burden appeared to decrease (Fig. 1a, b). The number of patients evaluated at each time point by PCM item is shown in Supplemental Table S1. The estimated decrease in risk per month that patients would develop moderate to severe symptoms after diagnosis was $5 \%$ $(P=0.02)$ for loss of interest in others, $1 \%(P=0.04)$ for trouble sleeping, and $1 \%(P=0.03)$ for anxiety. For the other symptoms, estimated risk of developing moderate to severe symptoms after bone metastases was found to increase over time (fatigue, $2 \%, P<0.001$; pain, $0.8 \%, P=0.09$; numbness and tingling, $0.3 \%, P=0.74$ ).

Using Kaplan-Meier methodology, the proportion of patients with moderate to severe symptoms over time after the onset of bone metastases (defined as 1 month prior to diagnosis of bone metastasis) was estimated (Fig. 2). Patients who already had the symptom at moderate to severe intensity at the onset of bone metastases are included in the analysis, and this proportion is represented at time zero. Pain and fatigue were the earliest symptoms to reach their median time to moderate/ severe intensity, which occurred within 1 month or less after the onset of bone metastases (Fig. 2a, b) and affected a majority of patients by 3 months after onset of bone metastases. Moderate to severe trouble sleeping and numbness/tingling commenced later, at a median 2.9 and 7.7 months, respectively, and also affected the majority of patients by the 1-year time point (Fig. 2c, d). Median time to moderate/severe anxiety was 14.7 months, and about half of the patients had this symptom at 1 year (Fig. 2e). Median time to loss of interest in others was not reached, and this symptom affected fewer patients (Fig. 2f).

Cox proportional hazards models were used to evaluate the impact of specific factors defined a priori associated with moderate/severe symptoms for each of the six variables. Baseline factors in the model included race, age, visceral metastases, bone-targeted agent treatment, prior taxane therapy, chemotherapy, brain metastases, liver metastases, and lung metastases. Fatigue was associated with chemotherapy use (HR 1.3; $95 \%$ CI 1.1, 1.5) and prior taxane therapy (HR
$1.3 ; 95 \%$ CI $1.1,1.7)$. The presence of visceral metastases (HR 1.3; $95 \%$ CI 1.0, 1.6) was a factor significantly associated with pain. Trouble sleeping was associated with age (HR 0.99 ; $95 \%$ CI $0.98,1.0$ ). For anxiety, significant factors included African-American race (compared with Caucasian; HR 0.6; $95 \%$ CI 0.4, 0.7), age (HR 0.99; $95 \%$ CI 0.98, 1.0), and presence of visceral metastases (HR 1.5; $95 \% \mathrm{CI}$ 1.1, 2.1). Numbness/tingling was associated with AfricanAmerican race (compared with Caucasian; HR 1.3; $95 \% \mathrm{CI}$ $1.1,1.6$ ), age (HR $0.99 ; 95 \%$ CI $0.98,1.0$ ), and chemotherapy (HR 1.3; $95 \%$ CI 1.1, 1.6), although the association with prior taxane therapy did not reach significance (HR 1.3; $95 \% \mathrm{CI}$ $0.98,1.6$ ). The site of metastases (lung/liver/brain) did not have significant effects on time to development of moderate or severe symptoms after the diagnosis of bone metastases.

\section{Discussion}

Numerous studies have reported symptom trajectories in patients with breast and other cancers relative to specific events such as initial diagnosis, surgery, chemotherapy, and death [10, 20-28]. The present study contributes to the understanding of symptom trajectory in the context of the onset and diagnosis of bone metastatic disease, showing that both the severity of symptoms as well as the proportion of patients experiencing moderate to severe symptoms began to increase for several months prior to the actual diagnosis of bone metastases. In particular, the risk of developing moderate or severe fatigue, pain, and numbness/tingling increased significantly in the months leading up to the diagnosis. The clinical implications of these findings are that oncologists should be aware that increasing symptoms in patients with advanced breast cancer may predict or reflect the onset of bone metastases. Earlier detection and treatment of bone metastases has the potential to reduce the increase of pain as well as to prevent skeletal events and loss of mobility incurred from bone destruction when bone metastases are discovered at a later stage.

Notably, after the diagnosis of bone metastases, the burden of fatigue, pain, and anxiety appeared to decrease or flatten, likely reflecting the effects of more aggressive patient management [6] which included introduction or intensification of pain medication, as well as the use of bone-targeted agents. A large longitudinal study in a mixed cancer population also reported a relatively flat burden of pain, nausea, anxiety, and depression in the 6 months before death, and the authors suggested that these symptoms are those for which medication strategies exist, although they were unable to evaluate treatment [27]. Alternatively, the decrease in PCM scores after bone metastases diagnosis in our study might have been influenced by the effect of missing data due to patient attrition and/or might reflect patient adjustment to progressive disease. 
The potential effects of increased patient management following diagnosis were also reflected in the Kaplan-Meier curves for all of the symptoms (except for loss of interest in others, a measure of depression), which showed a change in trend at 30 days after onset of bone metastases, with acceleration leading into the 30-day point and subsequent deceleration. The cumulative proportion of patients with moderate to severe symptoms increased over time for most symptoms, particularly for pain and fatigue which affected a majority of patients within 3 months of the onset of bone metastases. Trouble sleeping and numbness/tingling had slower onsets, but also affected a majority of patients at a moderate to severe intensity within 1 year after the onset. Anxiety and loss of interest in others developed in smaller proportions of patients, with a slower course than the other symptoms.

Previous studies of longitudinal symptom burden in our selected population, breast cancer patients with bone metastases, have reported increases of symptom burden over time, but either examined symptom burden as a composite [12], rather than individual symptoms, or focused on predictors of increased symptoms [13]. The study most similar to ours evaluated symptom trajectory in the 6 months before death in a mixed cancer population using the Edmonton Symptom Assessment System [27]. These authors demonstrated that fatigue, appetite, well-being, and drowsiness increased steadily in severity leading up to death, while pain, shortness of breath, anxiety, depression, and nausea remained relatively flat during this time period. In our study, we selected the group of symptoms identified in the ECOG SOAPP study as the most prevalent moderate to severe symptoms [7] in patients with breast cancer (fatigue/tiredness, disturbed sleep, pain, dry mouth, distress, numbness/tingling, and sadness) for which there were corresponding PCM items: fatigue, trouble sleeping, pain, anxious (as a measure of distress), numbness/ tingling, and loss of interest in others (as a measure depression [18]). In addition to the differences in individual symptoms selected for analysis in our study relative to previous studies, our results offer a unique perspective on the trajectory of symptoms owing to the anchoring of our analysis to the onset and diagnosis bone metastases.

Despite the decrease in pain after diagnosis of bone metastases, a substantial increase in analgesic use after bone metastases diagnosis was not observed in the present study, with just $16 \%$ of patients having new or increased pain medication after diagnosis. A major limitation to this dataset is that analgesic medication may have been provided by clinicians outside of the oncology practice. The proportion of patients in this study receiving opioid medications in the period leading up to diagnosis of bone metastases was approximately $10 \%$. This percentage is similar, although slightly lower, than the percentages of strong opioid use reported in an observational study evaluating the prescription of analgesics in patients with solid tumors (13\%) [29] and for patients with breast cancer and bone disease enrolled in a phase 3 clinical trial (16\%) [30].

The decrease in pain observed after the diagnosis of bone metastases may be associated with the use of bone-targeted agents and/or external beam radiation to manage pain without use of opioid analgesics $[2,4,30,31]$. Nearly half of the patients were receiving bone-targeted agents and these agents were prescribed rapidly following the diagnosis of bone metastases. We note that the data for bone-targeted agents are far more robust than for pain medication, as the former medications were administered in the oncologist's office and were therefore far more likely to be recorded. The observation that half of the patients did not receive any treatment with bonetargeted agents points to an opportunity to further improve management of bone metastases in this population.

A unique aspect of this dataset was that patient-reported outcomes were captured as part of routine assessment in the clinical practice setting, thereby allowing symptom burden to be studied over time relative to the diagnosis of bone metastases in the absence of a clinical trial. In addition, many patients included in this study would otherwise have been excluded from a prospective clinical trial due to low ECOG performance scores and/or limited life expectancy. Our study describes real-world findings in a population of over 1000 women treated in clinical practice across the USA that is representative of women treated in the community setting and therefore more generalizable to the broader breast cancer population of the USA than the population studied in clinical trials. Of note, the median time to diagnosis of bone metastases was 20 months in this population (in which half of the patients had stage IV disease), shorter than that recently reported for patients with local (stages 0 -II) or regional disease (stage III) (30 and 33 months, respectively) [32].

A strength of our study was the use of electronic medical records; however, electronic datasets in the clinical practice setting may also present some limitations as their use is still evolving and validation studies of the sensitivity and specificity of diagnostic and treatment data are needed. Importantly, these data reflect only diagnoses and treatments provided in the oncology clinic from community-based practices. Although close to $90 \%$ of community-based oncology/ hematology practices today have adopted electronic medical records for the routine care of patients [33], during the study period, the adoption of electronic health records was lower and therefore our study may differ in practice patterns and patient characteristics from the general US patient population. The identification of patients with bone metastases reliant on ICD-9 code in the OSCER database might be a source of misclassification and selection bias; selection bias is also possible from the inclusion criterion requiring at least one PCM assessment. Furthermore, analyses of pain medication use were limited by missing data. Drugs that are administered outside of the oncology clinic including oral medications 
and infusions) may not be recorded in the patient health record. These missing data are particularly important to the analyses of pain medication, as a majority are oral and many can be purchased over the counter at retail pharmacies. Therefore, we cannot exclude the possibility of underestimation of the pain medication use in this study, which may translate into the underestimation of the pain burden associated with the development of bone metastases in breast cancer patients. There are also other medications that could affect pain in cancer patients with bone metastases, such as bonetargeted agents, corticosteroids, and anticonvulsants, which may have confounded pain estimation. An additional limitation to this study is that site selection was not based on a random process since the data housed in OSCER with corresponding PCM data were limited to the Vector Oncology data from selected hematology/oncology practices.

In conclusion, we observed increasing symptom burden, particularly for pain and fatigue, both before and after the diagnosis of bone metastases in women with breast cancer in this retrospective patient record study conducted in the USA. These findings reinforce the need for symptoms to be proactively monitored and managed by health care providers. Advanced detection and treatment of bone metastases could reduce symptom burden and prevent further decline in HRQoL.

Acknowledgments This work was supported by Amgen Inc. The sponsor was involved in the study design; in the collection, analysis, and interpretation of data; in the writing of the report; and in the decision to submit the article for publication. Emma Booth of Amgen (Europe) $\mathrm{GmbH}$ and Wanda Krall, $\mathrm{PhD}$, on behalf of Amgen, provided assistance in the writing of this manuscript.

\section{Compliance with ethical standards}

Conflict of interest Charles Cleeland is a consultant of Astra Zeneca, Abbott, Genentech, Amgen, BMS, Pfizer, Astellas, Bayer, Actelion, J \& J, Novartis, Millennium, and Exelis. In addition, Dr. Cleeland has a patent for MDASI-MD Anderson Symptom Inventory-licensed to MD Anderson Cancer Center and himself, a patent for BPI-Brief Pain Inventory, and a patent for Cancer Symptom Science-Cambridge University Press with royalties paid.

Roger von Moos received a research grant from Amgen, Roche, and Merck and consulting fees from Amgen, BMS, GSK, Merck, Novartis, and Roche.

Mark Walker contracted research with Amgen, Genentech, Novartis, GSK, Lilly, and Onyx and consulting fees from Merck.

Yuanyuan Wang and Jianqing Gao have nothing to disclose.

Mariana Chavez-MacGregor is a consultant of Amgen, Novartis, Roche, and Genomic Health.

Alexander Liede, Jorge Arellano, Arun Balakumaran, and Yi Qian have employment and ownership interest in Amgen.

Open Access This article is distributed under the terms of the Creative Commons Attribution-NonCommercial 4.0 International License (http:// creativecommons.org/licenses/by-nc/4.0/), which permits any noncommercial use, distribution, and reproduction in any medium, provided you give appropriate credit to the original author(s) and the source, provide a link to the Creative Commons license, and indicate if changes were made.

\section{References}

1. Coleman RE (2006) Clinical features of metastatic bone disease and risk of skeletal morbidity. Clinical Can Res 12:6243s-6249s

2. Body JJ, Diel IJ, Bell R, Pecherstorfer M, Lichinitser MR, Lazarev AF, Tripathy D, Bergstrom B (2004) Oral ibandronate improves bone pain and preserves quality of life in patients with skeletal metastases due to breast cancer. Pain 111:306312

3. von Moos R, Body JJ, Egerdie B, Stopeck A, Brown JE, Damyanov D, Fallowfield LJ, Marx G, Cleeland CS, Patrick DL, Palazzo FG, Qian Y, Braun A, Chung K (2013) Pain and healthrelated quality of life in patients with advanced solid tumours and bone metastases: integrated results from three randomized, doubleblind studies of denosumab and zoledronic acid. Support Care Cancer 21:3497-3507

4. Wardley A, Davidson N, Barrett-Lee P, Hong A, Mansi J, Dodwell D, Murphy R, Mason T, Cameron D (2005) Zoledronic acid significantly improves pain scores and quality of life in breast cancer patients with bone metastases: a randomised, crossover study of community vs hospital bisphosphonate administration. Br J Cancer 92:1869-1876

5. Chow E, Hruby G, Davis L, Holden L, Schueller T, Wong R, Hayter C, Szumacher E, Loblaw A, Danjoux C (2004) Quality of life after local external beam radiation therapy for symptomatic bone metastases: a prospective evaluation. Support Cancer Ther 1:179-184

6. Cleeland CS (2006) The measurement of pain from metastatic bone disease: capturing the patient's experience. Clinical Can Res 12: 6236s-6242s

7. Cleeland CS, Zhao F, Chang VT, Sloan JA, O'Mara AM, Gilman PB, Weiss M, Mendoza TR, Lee JW, Fisch MJ (2013) The symptom burden of cancer: evidence for a core set of cancer-related and treatment-related symptoms from the Eastern Cooperative Oncology Group Symptom Outcomes and Practice Patterns study. Cancer 119:4333-4340

8. Kenne Sarenmalm E, Browall M, Gaston-Johansson F (2014) Symptom burden clusters: a challenge for targeted symptom management. A longitudinal study examining symptom burden clusters in breast cancer. J Pain Symptom Manage 47:731-741

9. Reed E, Simmonds P, Haviland J, Corner J (2012) Quality of life and experience of care in women with metastatic breast cancer: a cross-sectional survey. J Pain Symptom Manag 43:747-758

10. Stanton AL, Wiley JF, Krull JL, Crespi CM, Hammen C, Allen JJ, Barron ML, Jorge A, Weihs KL (2015) Depressive episodes, symptoms, and trajectories in women recently diagnosed with breast cancer. Breast Cancer Res Treat 154:105-115

11. Mendoza TR, Zhao F, Cleeland CS, Wagner LI, Patrick-Miller LJ, Fisch MJ (2013) The validity and utility of the M. D. Anderson Symptom Inventory in patients with breast cancer: evidence from the symptom outcomes and practice patterns data from the Eastern Cooperative Oncology Group. Clin Breast Cancer 13:325-334

12. Walker MS, Hasan M, Yim YM, Yu E, Stepanski EJ, Schwartzberg LS (2011) Retrospective study of the effect of disease progression on patient reported outcomes in HER-2 negative metastatic breast cancer patients. Health Qual Life Outcomes 9:46

13. Palesh OG, Collie K, Batiuchok D, Tilston J, Koopman C, Perlis ML, Butler LD, Carlson R, Spiegel D (2007) A longitudinal study of depression, pain, and stress as predictors of sleep disturbance among women with metastatic breast cancer. Biol Psychol 75:37-44 
14. Walker MS, Miller PJ, Namjoshi M, Houts AC, Stepanski EJ, Schwartzberg LS (2013) Relationship between incidence of fracture and health-related quality-of-life in metastatic breast cancer patients with bone metastases. J Med Econ 16:179-189

15. Lau EC, Mowat FS, Kelsh MA, Legg JC, Engel-Nitz NM, Watson HN, Collins HL, Nordyke RJ, Whyte JL (2011) Use of electronic medical records (EMR) for oncology outcomes research: assessing the comparability of EMR information to patient registry and health claims data. Clin Epidemiol 3:259-272

16. Abernethy AP, Zafar SY, Uronis H, Wheeler JL, Coan A, Rowe K, Shelby RA, Fowler R, Herndon JE 2nd (2010) Validation of the Patient Care Monitor (Version 2.0): a review of system assessment instrument for cancer patients. J Pain Symptom Manag 40:545-558

17. Fortner B, Baldwin S, Schwartzberg L, Houts AC (2006) Validation of the Cancer Care Monitor items for physical symptoms and treatment side effects using expert oncology nurse evaluation. J Pain Symptom Manag 31:207-214

18. Fortner B, Okon T, Schwartzberg L, Tauer K, Houts AC (2003) The Cancer Care Monitor: psychometric content evaluation and pilot testing of a computer administered system for symptom screening and quality of life in adult cancer patients. J Pain Symptom Manag 26:1077-1092

19. Mathias SD, Crosby RD, Qian Y, Jiang Q, Dansey R, Chung K (2011) Estimating minimally important differences for the worst pain rating of the Brief Pain Inventory-Short Form. J Support Oncol 9:72-78

20. Ancoli-Israel S, Liu L, Rissling M, Natarajan L, Neikrug AB, Palmer BW, Mills PJ, Parker BA, Sadler GR, Maglione J (2014) Sleep, fatigue, depression, and circadian activity rhythms in women with breast cancer before and after treatment: a 1-year longitudinal study. Support Care Cancer 22:2535-2545

21. Avis NE, Levine BJ, Case LD, Naftalis EZ, Van Zee KJ (2015) Trajectories of depressive symptoms following breast cancer diagnosis. Cancer Epidemiol Biomark Prev 24:1789-1795

22. Cheung WY, Barmala N, Zarinehbaf S, Rodin G, Le LW, Zimmermann C (2009) The association of physical and psychological symptom burden with time to death among palliative cancer outpatients. J Pain Symptom Manag 37:297-304

23. Fiszer C, Dolbeault S, Sultan S, Bredart A (2014) Prevalence, intensity, and predictors of the supportive care needs of women diagnosed with breast cancer: a systematic review. Psychooncology 23:361-374

24. Huang HP, Chen ML, Liang J, Miaskowski C (2014) Changes in and predictors of severity of fatigue in women with breast cancer: a longitudinal study. Int J Nurs Stud 51:582-592

25. Jones JM, Olson K, Catton P, Catton CN, Fleshner NE, Krzyzanowska MK, McCready DR, Wong RK, Jiang H, Howell D (2015) Cancer-related fatigue and associated disability in posttreatment cancer survivors. J Cancer Surviv. doi:10.1007/s11764015-0450-2

26. Sanford SD, Beaumont JL, Butt Z, Sweet JJ, Cella D, Wagner LI (2014) Prospective longitudinal evaluation of a symptom cluster in breast cancer. J Pain Symptom Manag 47:721-730

27. Seow H, Barbera L, Sutradhar R, Howell D, Dudgeon D, Atzema C, Liu Y, Husain A, Sussman J, Earle C (2011) Trajectory of performance status and symptom scores for patients with cancer during the last six months of life. J Clin Oncol 29:1151-1158

28. Trudel-Fitzgerald C, Savard J, Ivers H (2013) Evolution of cancerrelated symptoms over an 18-month period. J Pain Symptom Manag 45:1007-1018

29. Fisch MJ, Lee JW, Weiss M, Wagner LI, Chang VT, Cella D, Manola JB, Minasian LM, McCaskill-Stevens W, Mendoza TR, Cleeland CS (2012) Prospective, observational study of pain and analgesic prescribing in medical oncology outpatients with breast, colorectal, lung, or prostate cancer. J Clin Oncol 30:1980-1988

30. Cleeland CS, Body JJ, Stopeck A, von Moos R, Fallowfield L, Mathias SD, Patrick DL, Clemons M, Tonkin K, Masuda N, Lipton A, de Boer R, Salvagni S, Oliveira CT, Qian Y, Jiang Q, Dansey R, Braun A, Chung K (2013) Pain outcomes in patients with advanced breast cancer and bone metastases: results from a randomized, double-blind study of denosumab and zoledronic acid. Cancer 119:832-838

31. Cai B, Nickman NA, Gaffney DK (2013) The role of palliative external beam radiation therapy in boney metastases pain management. J Pain Palliat Care Pharmacother 27:28-34

32. Kremer R, Gagnon B, Meguerditchian AN, Nadeau L, Mayo N (2014) Effect of oral bisphosphonates for osteoporosis on development of skeletal metastases in women with breast cancer: results from a pharmaco-epidemiological study. J Natl Cancer Inst 106

33. Towle A, Godolphin W (2013) Patients as educators: interprofessional learning for patient-centred care. Med Teach 35:219-225 